(1)

CrossMark

\title{
Airway mucus accumulation in COPD: the cholinergic paradox!
}

\author{
Pierre-Régis Burgel (iD ${ }^{1,2}$
}

Affiliations: ${ }^{1}$ Institut Cochin, Université de Paris, INSERM U1016, Paris, France. ${ }^{2}$ Cochin Hospital, Dept of Respiratory Medicine, Assistance Publique Hôpitaux de Paris, Paris, France.

Correspondence: Pierre-Régis Burgel, Dept of Respiratory Medicine, Cochin Hospital, 27 rue du Faubourg Saint Jacques, 75014 Paris, France. E-mail: pierre-regis.burgeldaphp.fr

@ERSpublications

Cholinergic stimulation increases mucociliary clearance but long-acting anti-muscarinic treatment remains one of the major therapies for patients with COPD http://bit.ly/2RlXAwl

Cite this article as: Burgel P-R. Airway mucus accumulation in COPD: the cholinergic paradox! Eur Respir J 2020; 55: 1902473 [https://doi.org/10.1183/13993003.02473-2019].

Mucus accumulation, related to excess mucus production and secretion in proximal and distal airways, is an established characteristic in the airways of patients with COPD. Chronic bronchitis, a consequence of cigarette smoking that can be found in smokers with or without COPD, is a disabling symptom and has been associated with increased rates of exacerbations and hospitalisations in patients with COPD [1] and in subjects with normal spirometry [2]. Chronic bronchitis is usually ascribed to inflammation and airway mucus hypersecretion by airway epithelium and submucosal glands in proximal airways [3]. In distal airways, where submucosal glands are absent, airway mucus is believed to derive from airway epithelium and airway mucus plugging has been associated with airflow limitation [4] and survival [5]. Although targeting mucus accumulation could represent a therapeutic option in patients with COPD, current drugs have only limited effects on excess mucus production, secretion and/or clearance [6]. Difficulties in making progress in this area have included the absence of appropriate animal models (as rodents have few airway submucosal glands) and a complexity in studying regulation of mucus production, composition of airway mucus and mucociliary clearance. Development of novel animal models in larger animals, in which the airway structure is more similar to that in humans, and progress in the tools for studying mucus contents and mucociliary clearance, has led to recent progress in our understanding of this major feature of chronic airway diseases [7].

In the present issue of the European Respiratory Journal, Lin et al. [8] present novel and interesting data on airway mucus clearance in COPD. The authors used a combination of animal studies in cigarette smoke exposed ferrets and human studies in cultured airway epithelial cells obtained from patients with COPD. The authors first showed that in vivo mucociliary clearance measured by Tc-DTPA retention is reduced in ferrets exposed to cigarette smoke for 6 months as compared to air controls. Using micro-optical coherence tomography to analyse excised ferret trachea, the authors confirmed a reduction by approximately $30 \%$ in mucociliary transport in animal exposed to cigarette smoke. These findings were not associated with significant differences in airway hydration or ciliary beating frequency, whereas experiments in cultured human airway epithelial cells (which contained no gland) showed that cigarette smoke exposure for $24 \mathrm{~h}$ was associated with reduced mucus hydration (as shown by reduced airway surface liquid depth), reduced ciliary beating frequency and reduced mucociliary transport. Nevertheless, independent predictors of mucociliary transport in the excised ferret trachea included smoking exposure, airway surface liquid depth and ciliary beating frequency. The authors then explored the effects of 
cholinergic stimulation by carbachol in ferret and human airways. Carbachol treatment for $30 \mathrm{~min}$ increased airway surface liquid depth, ciliary beating frequency and mucociliary transport in excised tracheas from air- and smoke-exposed ferrets. The authors suggested that cholinergic stimulation can partially rescue defects in microanatomical function in smoking-related lung disease. Finally, histology in ferret and human excised airways confirmed the impact of cigarette-smoke on mucus accumulation. Mucus viscosity and solid contents were increased in cultured airway epithelium from COPD patients, as compared with smokers without COPD and non-smokers.

This study constitutes a major effort in the understanding of the complex regulation of airway mucus biology in the context of cigarette smoking/COPD. The authors should be congratulated for developing a unique animal model and for using state-of-the-art techniques to measure mucociliary transport and mucus structure in both human and animal airways. There were also limitations to the models analysed: the excised ferret trachea contains submucosal glands, an obvious advantage compared to culture airway epithelial cells, because airway mucus in proximal airways is derived from both glands and airway epithelium. However, it provides no information on distal airways, the major site of airflow limitation in patients with COPD [4]. Short-term $(30 \mathrm{~min})$ treatment of excised trachea with carbachol allowed demonstration of improvement in mucus hydration and mucociliary transport after cholinergic stimulation, but did not allow measurement of longer effects of cholinergic stimulation in airways. Mechanisms leading to mucus accumulation in airways are complex, and include not only reduced mucociliary transport but also increased mucin production and secretion [6], and/or reduced mucin degradation within the airways [9]. The present study focused on mucus transport, which does not account for multiple mechanisms responsible for mucus accumulation in airways.

Findings that diminished mucus transport is a major consequence of smoke exposure, and is impacted by airway dehydration, ciliary beating and mucus viscosity are important, but therapeutic application of the present studies remains to be established. Perhaps the most questionable issue relates to the proposal of increasing cholinergic stimulation in the airways of patients with COPD. Increased cholinergic tone from both neuronal and non-neuronal systems are well-established features in the airways of patients with COPD [10, 11]. Antimuscarinic receptor antagonists are one of the main treatments in patients with COPD [12]. Previous studies have shown that carbachol induced mucin synthesis in human bronchial tissue and in cultured airway epithelium [13], suggesting that cholinergic stimulation may have deleterious effects on airway mucus accumulation in humans. In vitro and rodent studies have further shown that antimuscarinic receptor antagonists reduced mucus accumulation triggered by noxious stimuli (e.g. cigarette smoke, neutrophil elastase or lipopolysaccharide) in airways [6], a mechanism that may be related to reduction in epidermal growth factor receptor-mediated airway mucin production. In the present report, LiN et al. [8] suggested that activating the nicotinic pathway (independently of treatment with the muscarinic receptor antagonist) may be explored for improving mucus hydration in patients with COPD [8]. However, Chen et al. [14] reported that exposure of cultured airway epithelial cells to nicotine lead to reduced hydration of mucins and increased mucus viscosity. Thus, it remains unclear whether selectively activating the cholinergic pathway would result in clinical benefit in patients with COPD.

Targeting excess mucus accumulation in the airways of patients with chronic airway diseases (e.g. chronic bronchitis and/or COPD) remains presumably an important therapeutic approach. Increasing mucus transport and clearance by targeting ion transport in the airways with the goals of improving airway dehydration and reducing mucus viscosity is an attractive idea. However, the idea of increasing, even selectively, the cholinergic pathway in patients with COPD appears questionable, and may be viewed as the "cholinergic paradox". Cholinergic tone is already increased in patients with COPD and antimuscarinic agents have beneficial effects, which are likely in part mediated by their effect on reducing mucin production in airway epithelium. Alternative approaches suggested by the authors (e.g. activation or potentiation of the ion channels' cystic fibrosis conductance regulator [15] and/or the epithelial sodium channel ENaC [16]) may be better suited to attenuate mucus clearance defects in patients with COPD. It remains an exciting time in this field, where old ideas have the potential to be tested in novel in vitro and animal models, as in the study by LIN et al. [8], and where new molecules are developed that will ultimately be tested in humans!

Conflict of interest: P-R. Burgel reports personal fees from AstraZeneca, Boehringer Ingelheim, Chiesi, GSK, Novartis, Pfizer, Vertex, Teva, Zambon and Mylan, outside the submitted work.

\section{References}

1 Burgel PR, Nesme-Meyer P, Chanez P, et al. Cough and sputum production are associated with frequent exacerbations and hospitalizations in COPD subjects. Chest 2009; 135: 975-982. 
2 Colak Y, Nordestgaard BG, Vestbo J, et al. Prognostic significance of chronic respiratory symptoms in individuals with normal spirometry. Eur Respir J 2019; 54: 1900734.

3 Mullen JB, Wright JL, Wiggs BR, et al. Reassessment of inflammation of airways in chronic bronchitis. $\mathrm{Br} \mathrm{Med} J$ 1985; 291: 1235-1239.

4 Hogg JC, Chu F, Utokaparch S, et al. The nature of small-airway obstruction in chronic obstructive pulmonary disease. $N$ Engl J Med 2004; 350: 2645-2653.

5 Hogg JC, Chu FS, Tan WC, et al. Survival after lung volume reduction in chronic obstructive pulmonary disease: insights from small airway pathology. Am J Respir Crit Care Med 2007; 176: 454-459.

6 Martin C, Frija-Masson J, Burgel PR. Targeting mucus hypersecretion: new therapeutic opportunities for COPD? Drugs 2014; 74: 1073-1089.

$7 \quad$ Boucher RC. Muco-obstructive lung diseases. N Engl J Med 2019; 380: 1941-1953.

8 Lin VY, Kaza N, Birket SE, et al. Excess mucus viscosity and airway dehydration impact COPD airway clearance. Eur Respir J 2020; 55: 1900419.

9 Innes AL, Carrington SD, Thornton DJ, et al. Ex vivo sputum analysis reveals impairment of protease-dependent mucus degradation by plasma proteins in acute asthma. Am J Respir Crit Care Med 2009; 180: 203-210.

10 Montalbano AM, Di Sano C, Chiappara G, et al. Cigarette smoke and non-neuronal cholinergic system in the airway epithelium of COPD patients. J Cell Physiol 2018; 233: 5856-5868.

11 Barnes PJ. The role of anticholinergics in chronic obstructive pulmonary disease. Am J Med 2004; 117: Suppl. $12 \mathrm{~A}, 24 \mathrm{~s}-32 \mathrm{~s}$.

12 Beeh KM, Burgel PR, Franssen FME, et al. How do dual long-acting bronchodilators prevent exacerbations of chronic obstructive pulmonary disease? Am J Respir Crit Care Med 2017; 196: 139-149.

13 Cortijo J, Mata M, Milara J, et al. Aclidinium inhibits cholinergic and tobacco smoke-induced MUC5AC in human airways. Eur Respir J 2011; 37: 244-254.

14 Chen EY, Sun A, Chen CS, et al. Nicotine alters mucin rheological properties. Am J Physiol Lung Cell Mol Physiol 2014; 307: L149-L157.

15 Solomon GM, Fu L, Rowe SM, et al. The therapeutic potential of CFTR modulators for COPD and other airway diseases. Curr Opin Pharmacol 2017; 34: 132-139.

16 Astrand $\mathrm{AB}$, Hemmerling $\mathrm{M}$, Root $\mathrm{J}$, et al. Linking increased airway hydration, ciliary beating, and mucociliary clearance through ENaC inhibition. Am J Physiol Lung Cell Mol Physiol 2015; 308: L22-L32. 